\title{
Mesenchymal to amoeboid transition is associated with stem-like features of melanoma cells
}

\author{
Maria Letizia Taddei ${ }^{1}$, Elisa Giannoni ${ }^{1}$, Andrea Morandi ${ }^{1}$, Luigi Ippolito ${ }^{1}$, Matteo Ramazzotti ${ }^{1}$, Maurizio Callari ${ }^{2}$, \\ Paolo Gandellini ${ }^{2}$ and Paola Chiarugi $i^{1,3^{*}}$
}

\begin{abstract}
Background: Cellular plasticity confers cancer cells the ability to adapt to microenvironmental changes, a fundamental requirement for tumour progression and metastasis. The epithelial to mesenchymal transition (EMT) is a transcriptional programme associated with increased cell motility and stemness. Besides EMT, the mesenchymal to amoeboid transition (MAT) has been described during tumour progression but to date, little is known about its transcriptional control and involvement in stemness. The aim of this manuscript is to investigate (i) the transcriptional profile associated with the MAT programme and (ii) to study whether MAT acquisition in melanoma cancer cells correlates with clonogenic potential to promote tumour growth.

Results: By using a multidisciplinary approach, we identified four different treatments able to induce MAT in melanoma cells: EphA2 overexpression, Rac1 functional inhibition using its RacN17 dominant negative mutant, stimulation with llomastat or treatment with the RhoA activator Calpeptin. First, gene expression profiling identified the transcriptional pathways associated with MAT, independently of the stimulus that induces the MAT programme. Notably, gene sets associated with the repression of mesenchymal traits, decrease in the secretion of extracellular matrix components as well as increase of cellular stemness positively correlate with MAT. Second, the link between MAT and stemness has been investigated in vitro by analysing stemness markers and clonogenic potential of melanoma cells undergoing MAT. Finally, the link between MAT inducing treatments and tumour initiating capability has been validated in vivo.

Conclusion: Taken together, our results demonstrate that MAT programme in melanoma is characterised by increased stemness and clonogenic features of cancer cells, thus sustaining tumour progression. Furthermore, these data suggest that stemness is not an exclusive feature of cells undergoing EMT, but more generally is associated with an increase in cellular plasticity of cancer cells.
\end{abstract}

Keywords: Melanoma, Cell plasticity, Amoeboid motility, Stemness, EphA2

\section{Background}

Plasticity in cell motility is a key prerequisite for the metastatic dissemination of tumour cells. Indeed, cancer cells may achieve different types of cell motility, including mesenchymal, collective, and amoeboid styles. Thus, by shifting between different motility styles, migrating cells can adapt to environmental changes and matrix stiffness to

\footnotetext{
*Correspondence: paola.chiarugi@unifi.it

'Department of Experimental and Clinical Biomedical Sciences, Tuscany Tumor Institute, University of Florence, viale Morgagni 50, Florence 50134, Italy

${ }^{3}$ Center for Research, Transfer and High Education 'Study at Molecular and Clinical Level of Chronic, Inflammatory, Degenerative and Neoplastic Disorders for the Development on Novel Therapies', Florence, Italy Full list of author information is available at the end of the article
}

elude anticancer treatments, which represents a major challenge for developing strategies aimed at blocking the dissemination of cancer cells [1-3]. Mesenchymal motility depends on extra-cellular matrix (ECM) proteolysis through production of matrix metalloproteinases (MMPs). It is characterised by activation of Rac1 at the leading edge of the cell, and inhibition of RhoA GTPase [3], conferring to migrating cells an elongated and polarized cell morphology. Conversely, amoeboid motility is characterised by squeezing movements that allow cancer cells to glide through matrix barriers, without the use of MMPs and integrin engagement. Amoeboid movement is characterised by a rounded morphology, high Rho kinase signalling to drive elevated levels of actomyosin 
contractility. MMP inhibitors have been tested clinically but failed to have effect on tumour metastasis [4], probably due to the plasticity of tumour cells and their ability to invade in an amoeboid manner in the absence of protease activity [5].

Previous data report that the epithelial mesenchymal transition (EMT) is promoted by the induction of a transcriptional programme that has been associated with the activation of several key transcriptions factors, including Snail (SNAI1), Slug (SNAI2), Twist and ZEB-1/2. This transcriptional programme ultimately leads to the disruption of adherens junctions, activation of polarized cell motility and increased degradation of ECM through secretion of MMPs [6-8]. In addition to EMT, a second type of motility shift has been described as essential in tumour progression, i.e., mesenchymal amoeboid transition (MAT) [9]. MAT can be induced in cancer cells by pharmacological inhibition of integrin function or MMP activity, by p53 or p27 deficiency [10,11], as well as through the activation or re-expression of EphA2 [5,12-14]. Although MAT confers a clear advantage to metastatic processes, very little is known about the molecular events that promote this motility shift $[12,13,15]$.

Mammary epithelial cells undergoing EMT are endowed with stem-cell features, generating anchorageindependent mammospheres, soft agar colonies, and tumours [16]. Accordingly, we previously reported that the contact with cancer associated fibroblasts promotes EMT in the neighbouring prostate carcinoma cells, allowing them to acquire stem cell traits [17]. On the other hand, nothing is known to our knowledge, about a possible transcriptional regulation of amoeboid motility. However, also for MAT we have recently discovered a possible link with stemness. Indeed, in prostate cancer and glioblastoma, EphA2 expression, which induces an amoeboid motility, has been associated with achievement of stemness markers, increased clonogenic potential and tumour growth $[15,18]$.

Melanoma cells are endowed with great plasticity in migration. Indeed, we have recently demonstrated that melanoma cells are able to shift between mesenchymal and amoeboid motility: melanoma cells move mesenchymally in response to pro-inflammatory cytokines, whereas after re-expression of embryonic EphA2 receptor, they achieve an amoeboid motility style giving rise to successful metastatisation [13]. Furthermore, Sanz-Moreno et al. showed that A375M2 primary melanoma cells can switch ad hoc between mesenchymal and amoeboid motility [19]. Furthermore, the same authors have recently demonstrated that treatment of melanoma cells with the Src inhibitor dasatinib results in a switch from mesenchymal migration to ROCK-dependent amoeboid invasion, confirming, once again, that cancer cell migratory capabilities could be blocked only by a combination of different treatments effective in the inhibition of both mesenchymal and amoeboid motility styles [20]. To confirm that cancer cells often undergo plasticity in cell motility, the opposite transition has been also described: the group of Marshall demonstrated that A375 M2 melanoma cells move in a rounded, amoeboid manner on top of or through collagen matrices due to JAK1-dependent MLC2 phosphorylation, whereas silencing of JAK1 induces a reduction in the acto-myosin contractility and the acquisition of an elongated morphology [21]. Moreover, the block of p53 function is sufficient to convert melanoma cells from an elongated motility style to a rounded locomotion, suggesting that such switch would favour the dissemination of p53-defective tumour cells by increasing their invasiveness [11].

In this light, the aim of our work is to investigate the regulation of mesenchymal to amoeboid transition induced in human melanoma cells by different stimuli and the possible link with the acquisition of clonogenic potential in order to sustain tumour growth in response to changes in microenvironmental conditions.

\section{Results and discussion}

EphA2 or RacN17 overexpression, treatment with Rho activator or ilomastat induces an amoeboid motility style in Hs294T melanoma cells

Previous studies from our laboratory demonstrated that overexpression of EphA2 in murine melanoma cells converts their migration style from mesenchymal to amoeboid like, thus conferring a cell plasticity in tumour invasiveness [13]. We now investigate the induction of an amoeboid motility style in human melanoma Hs294T cells following EphA2 overexpression and compare to amoeboid motility induced by RacN17 overexpression, treatment with the Rho activator Calpeptin or the MMPs inhibitor Ilomastat. We first analysed the activation level of RhoA and Rac1 small GTPases, as both RhoA activation and Rac1 inhibition have been correlated with a proteolysis independent motility style [12]. As shown in Figure 1A all these treatments are able to activate RhoA and to inhibit Rac1, thus suggesting a possible induction of an amoeboid motility in human melanoma cells. In addition, following all the aforementioned treatments, melanoma cells undergo cell rounding, a typical prerequisite for the acquisition of an amoeboid motility (Figure 1B). The confirmation that these cells undergo a real MAT emerges from the analysis of cell morphology in 3D collagen matrices, using confocal fluorescence reflection microscopy. As shown in Figure $1 \mathrm{C}$ all these treatments cause the acquisition of a round-shaped squeezing morphology while control cells maintain an elongated profile and establish contacts with collagen fibers. In addition, to exclude that the induction of the amoeboid morphology could be toxic for 

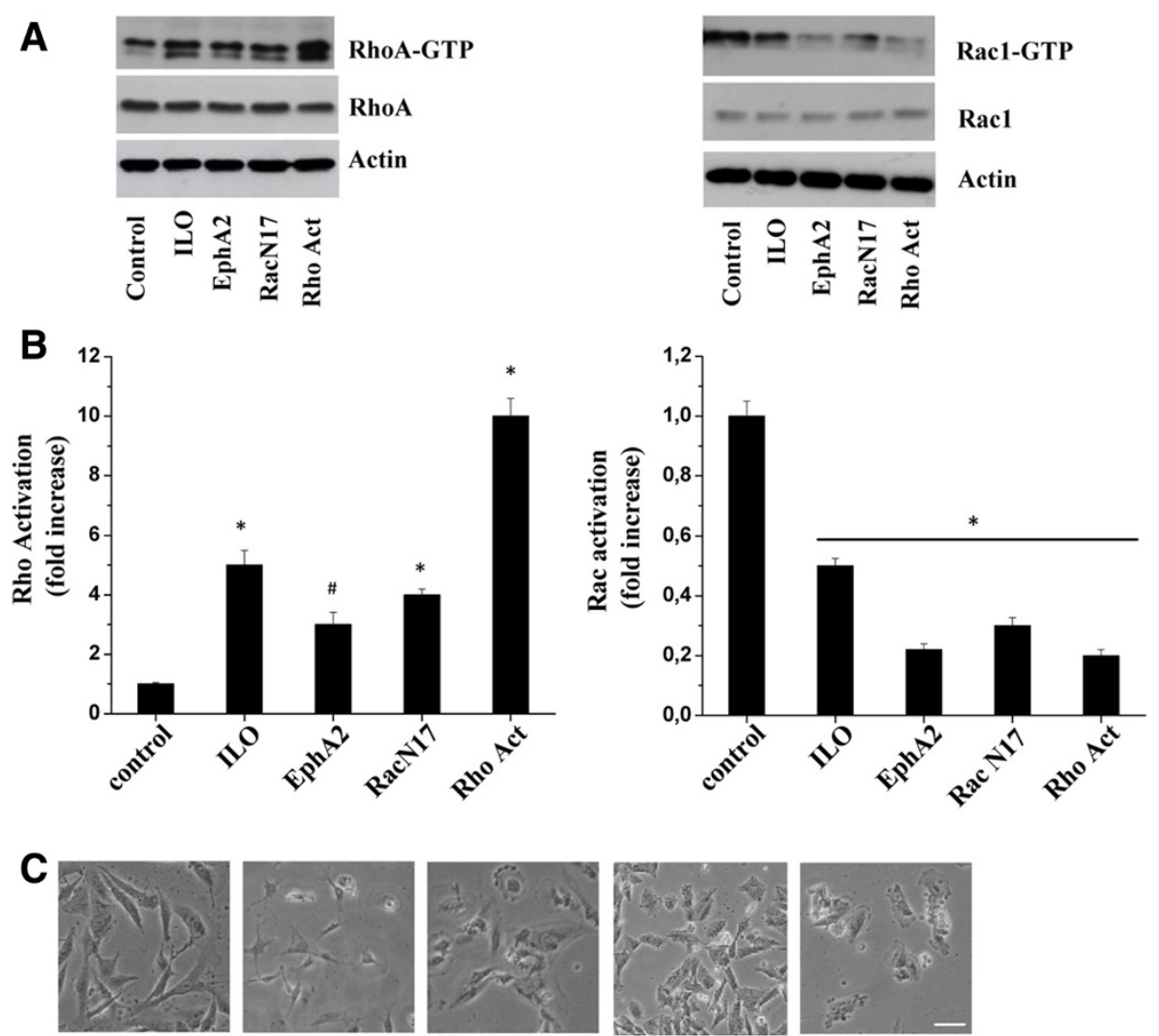

Control

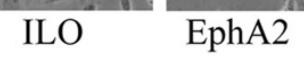

RacN17

Rho Act

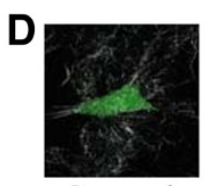

Control

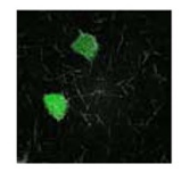

ILO

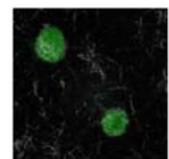

EphA2

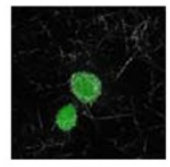

RacN17

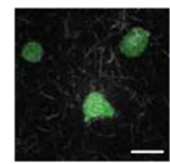

Rho Act

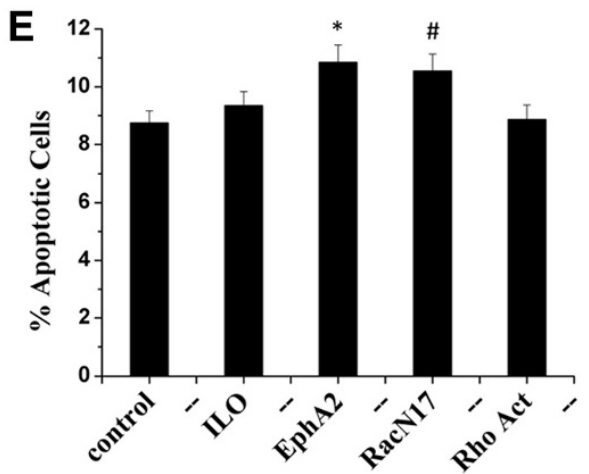

Figure 1 (See legend on next page.) 
(See figure on previous page.)

Figure 1 EphA2 or RacN17 overexpression, treatment with Rho activator or ilomastat induce RhoA activation and Rac1 inhibition and acquisition of a rounded morphology. A) $10^{6} \mathrm{Hs} 294 \mathrm{~T}$, EphA2 or RacN17 transfected cells were serum starved for 48 h, for llomastat treatment cells were serum starved for $48 \mathrm{~h}$ in the presence of $50 \mu \mathrm{mol} / \mathrm{L}$ llomastat, for Rho activation treatment cells were serum starved for $48 \mathrm{~h}$ and then stimulated with $1 \mathrm{U} / \mathrm{ml}$ Calpeptin for $2 \mathrm{~h}$ at $37^{\circ} \mathrm{C}$. After treatments, RhoA-GTP and Rac1-GTP were analysed by pull-down assay from cell lysates. The total amount of RhoA and Rac1 were quantified by anti-Rho and anti-Rac1 immunoblot. An anti-actin antibody was used to ensure equal protein loading. The bar graphs obtained from densitometry analysis of triplicate experiments are shown. Student t-test, ${ }^{*} p<0.001$ treatments vs control, \#p $<0.005$ treatments vs control. B) Cells were treated as in A), photographs were taken and a representative image of their morphology is shown. Bar, $20 \mu \mathrm{m}$. C) Cell treated as in A) were labeled with CFSE and then incubated in three-dimensional collagen lattice. Cell morphology was monitored by confocal fluorescence-reflection microscopy. Photographs are representative of several randomly chosen fields. Bar, $20 \mu \mathrm{m}$.

D) Cells were treated as in A). The percentage of apoptotic cells was evaluated using the Muse ${ }^{\text {TM }}$ Annexin V \& Dead Cell kit. The results are representative of three experiments with similar results. Student t-test, ${ }^{*} \mathrm{p}<0.01 \mathrm{EphA2}$ vs control, \#p $<0.05$ RacN17 vs control. cells, we performed a cell viability assay. Figure 1D shows that none of the aforementioned treatments interfere with cell viability. To further investigate the motility of EphA2 or RacN17 overexpressing cells, as well as cells treated with Rho activator or Ilomastat, we tested their invasive abilities to cross a Matrigel barrier in the presence or absence of the MMPs inhibitor, Ilomastat. Indeed, sensitivity to protease inhibition of invasive ability of cells has been widely used as a mesenchymal/amoeboid discriminant test $[13,15]$. As shown in Figure 2A, control cells behaviour is highly influenced by the presence of Ilomastat. Conversely, cells exposed to MAT inducing treatments are completely unaffected by the presence of Ilomastat, suggesting that they preferentially use a MMPs-independent motility (Figure 2A). Indeed, the acquisition of an amoeboid motility style has been confirmed by MMPs analysis by gelatine zymography, which actually reveals both a decrease in expression and activation of MMP2 in cells overexpressing EphA2, RacN17 or treated with Rho activator or Ilomastat (Figure 2B). Overall, these data confirm that Hs294T melanoma cells undergo a clear MAT in response to all treatments used, highlighting the great plasticity in cell motility of these tumour cells.

\section{EphA2 overexpression, treatment with Rho activator or ilomastat activate common signalling pathways to achieve amoeboid motility}

It is now well established that EMT is an epigenetic programme, mainly regulated at a transcriptional level, involving several factors, such as Snail, Slug, Twist, Goosecoid, ZEB1, and SIP1 [22,23]. We next investigated whether MAT induction displays common transcriptional traits independently of the stimuli that activate the MAT programme by gene expression profiling on Hs294T cells overexpressing EphA2, or treated with Ilomastat or the Rho activator Calpeptin. A class comparison was performed between treated cells and controls, ranking all genes according to Student t-test statistics. Gene set enrichment analysis (GSEA) [24] was applied to such ranked list to identify gene sets directly or inversely associated with MAT inducing treatments (i.e.
EphA2, Ilomastat and Rho activator). The GSEA analysis revealed that MAT induction, independently of the stimulus that has activated the MAT programme, associates with several biofunctions represented by multiple gene sets, as reported in Table 1 and Additional file 1: Table S1. Notably, GSEA analysis revealed that the activation of the MAT programme associates with the repression of features that are characteristics of cell undergoing EMT as shown by a negative correlation with ANASTOSIOU CANCER_MESENCHYMAL_TRANSITION_SIGNATURE gene set. However, a positive correlation was found with gene sets that are related to TGF- $\beta$, a well-known EMT inducer, targets of the EMT activator ZEB1 and with targets that are down regulated by E-cadherin expression (Table 1, epithelial mesenchymal transition). This let us speculate that MAT programme is not just a phenomenon that recapitulates the mesenchymal-to-epithelial transition (MET). The ability to acquire an amoeboid motility confers the cancer cells characteristics that are both of mesenchymal and epithelial cells and therefore are features of aggressive cancer cells with high plasticity. In keeping with the fact that MAT is significantly regulated by RhoA activation [12], we observed a positive correlation with the BERENJENO_TRANSFORMED_BY_RHOA_UP gene sets (Table 1, Rho). Based on our data, we suggest a model where EMT and MAT are different status that a cancer cell can display during cancer progression. Particularly, the EMT has to be at least partially repressed to allow the cell to enter into the MAT status, suggesting a hierarchy between EMT and MAT where MAT is a consecutive event of the EMT programme. Crucially, this may explain why clinical trials aimed at blocking EMT using anti proteolytic agents did not succeed $[4,5]$.

It is well known that MAT is promoted in looser matrices and is independent on contacts between cells and ECM [25-27]. In agreement with this rearrangement of the ECM, many of the gene sets identified positively correlate with a decrease in the secretion of ECM components, e.g. collagen, and with a reduction of contacts between cellular receptors (Table 1, extracellular matrix gene sets). These data suggest that MAT transcriptional 
A

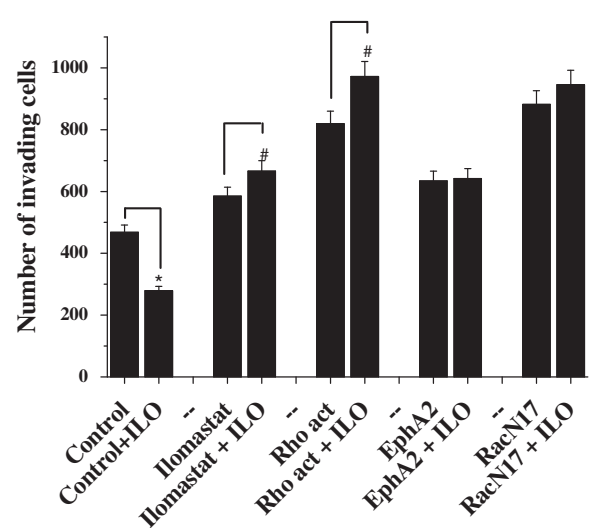

Control
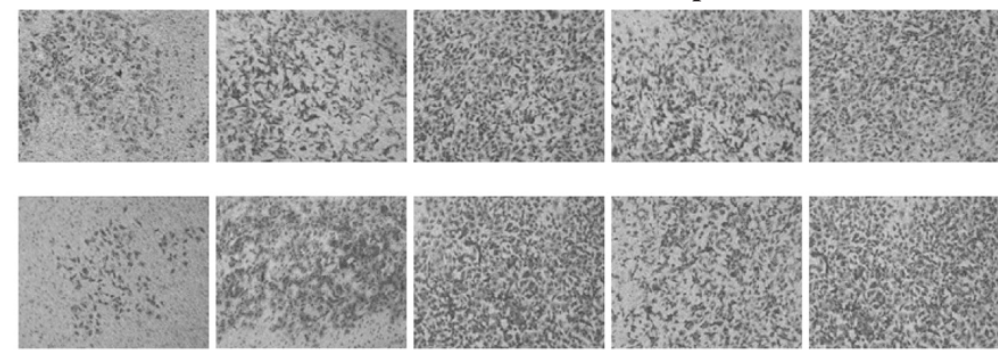

+ Ilomastat

B

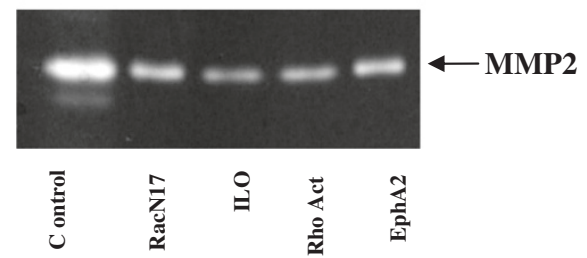

Figure 2 Cells overexpressing EphA2 or RacN17, treated with Rho activator or ilomastat acquire an amoeboid- MMP-independent motility style. A) Hs294T, EphA2 or RacN17 transfected cells were serum starved for 48 h, for llomastat treatment cells were serum starved for $48 \mathrm{~h}$ in the presence of $50 \mathrm{\mu mol} / \mathrm{L}$ llomastat, for Rho activation treatment cells were serum starved for $48 \mathrm{~h}$ and then stimulated with $1 \mathrm{U} / \mathrm{ml}$ Calpeptin for $2 \mathrm{~h}$ at $37^{\circ} \mathrm{C}$. Then, $6 \times 10^{4}$ cells were seeded into the upper compartment of Boyden chamber with or without $50 \mu \mathrm{M}$ llomastat. Cells were allowed to migrate through the filter coated with Matrigel toward the lower compartment filled with complete medium. Cell invasion was evaluated after Diff-Quick staining by counting cells in six randomly chosen fields. The results are representative of three experiments with similar results. Student t-test, ${ }^{*} p<0.001$ ILO treatment vs untreated, $\# p<0.05$ ILO treatment vs untreated. B) Analysis of MMP activity. Media from confluent monolayers of cells treated as in A) were collected and analysed by gelatine zymography. The clear bands represent areas of gelatinase activity. The results shown are representative of four experiments.

programme associates with a change of the matrix stiffness that supports amoeboid motility style. In fact, amoeboid moving cells that need to squeeze within ECM fibres do not rely on proteolytic degradation of robust ECM and would certainly benefit from the release of cell adhesion bindings, from loosen and relax ECM stiffness, as well as from changes in ECM composition.

Although we observed that EMT is impaired during MAT, some features of EMT, emerged also from GSEA analysis, are maintained in cells moving with amoeboid motility. Indeed, we observe that MAT inducers treatment of Hs294T cells positively associates with gene sets involved in anoikis resistance and cell survival such as Epidermal Growth Factor (EGF) and Neuregulin 1 (NRG1) (Table 1, EGF signalling). Anoikis resistance is an essential requirement for a cancer cell that leaves the primary site to survive in the blood stream in order to metastasize to distant sites. This ability is even more crucial for a cell that is moving with amoeboid motility, i.e. independently of integrin engagement [12]. A positive correlation of MAT inducers treatment of melanoma cells with EGF signalling in cells moving with amoeboid motility is in keeping with previous studies describing EGFR activation during protection from anoikis [28]. Indeed, cells can prevent anoikis through the oxidation/activation of the tyrosine kinase Src, thus granting 
Table 1 GSEA analysis: gene sets significantly correlated with MAT-inducing treatments

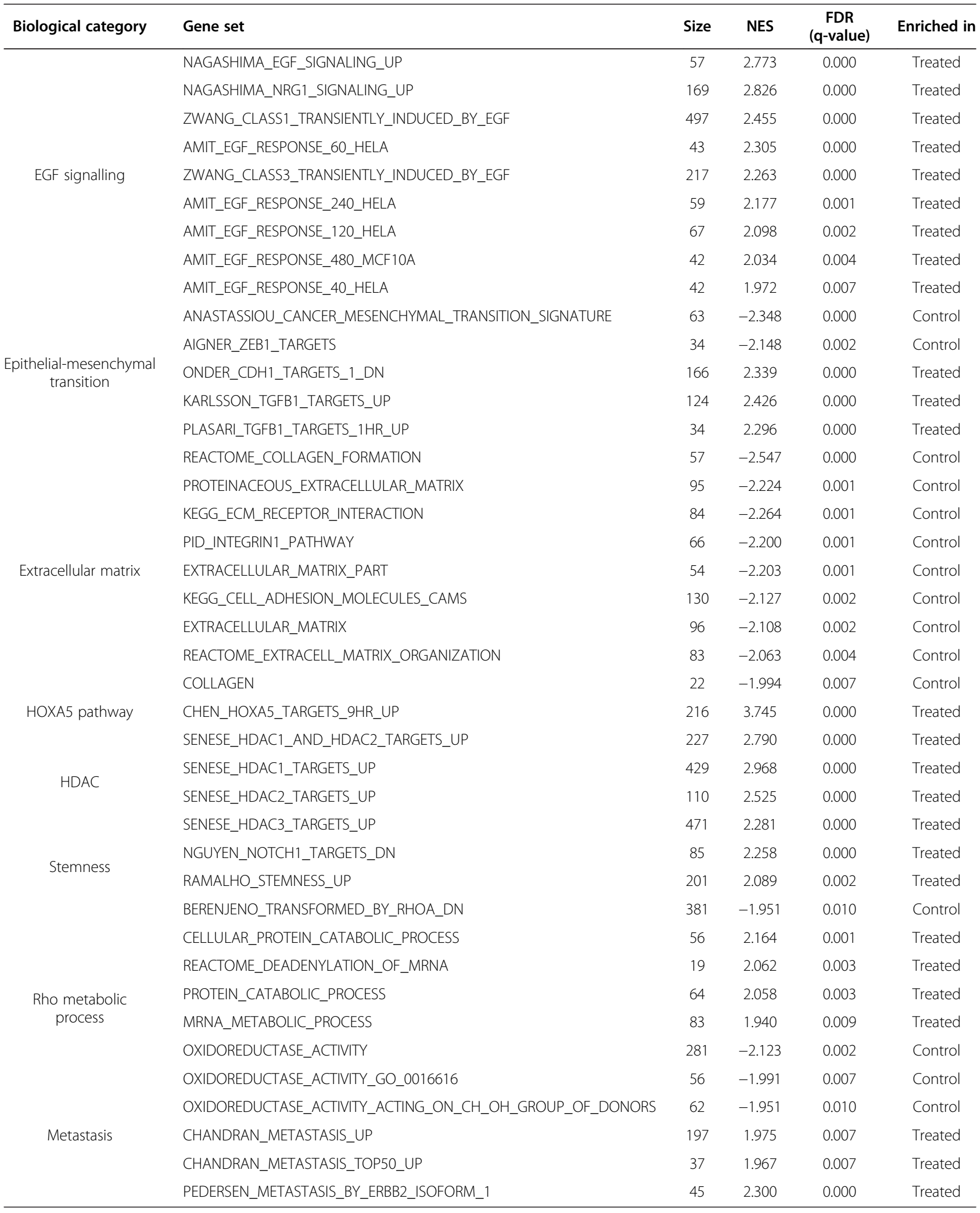


the activation of pro-survival pathways through a Srcdependent and ligand-independent phosphorylation of EGFR, which leads to Bim degradation [28].

It is now well established that EMT correlates with the achievement of stemness traits in multiple cancer models $[16,17]$. In addition, we have recently demonstrated that in prostate carcinoma cells, EphA2 silencing induces the loss of amoeboid motility style as well as a decrease in stem cell markers, thus suggesting that also MAT can be related to stemness and tumour growth [15]. In keeping with these observations, we observed that MAT inducing treatments in melanoma cells positively correlate with stemness gene sets (Table 1, stemness), suggesting that the achievement of stemness traits is not limited to EMT programme, but is a more general feature associated with the plasticity of tumour cell motility. These data suggest that, although EMT is a transcriptional programme leading to achievement of stemness traits $[16,17,29]$, the additional shift occurring in cancer cells undergoing MAT contributes and enhances these stem-like features, further promoting the spread of metastases. In keeping, GSEA analysis revealed that metastases associated gene sets positively correlates with MAT inducers treatment (Table 1, metastasis). Furthermore, following MAT induction in Hs294T cells, we observed (i) a positive correlation with gene sets related to protein catabolism and (ii) a negative correlation with anabolic processes (Table 1, metabolic process). The increase in catabolic processes, likely connected to autophagy and leading cancer cells to selfsustain their metabolism during starvation, is a very common feature of cancer cells. Indeed, several tumours are often exposed to oxygen or nutrient deprivation, owing to mass overgrowth and insufficient angiogenesis [30]. Engagement of self-cannibalism and autophagic strategies have been indicated as protective against environmental stress, nutrient deprivation or chemotherapy treatment [31-33]. Metabolic deregulation of cancer cells during tumour progression has now attracted the interest of oncologists and is now a new Hallmark of Cancer [34], but there are very few data describing the metabolic reprogramming of cancer cells upon changes in their motility styles to compare them with the output of our GSEA analysis. Interestingly, EMT has been correlated with enhancement of anabolic processes, increase in cell biomass and therefore in cancer growth. While MAT appears an exacerbation of EMT programme for several aspects (e.g. activation of survival pathways and achievement of stemlike traits), the metabolic features of EMT or MAT undergoing cells appear to diverge.

Similarly to what observed in EMT, we suppose that also for the MAT programme a typical transcriptional profile could be identified. MAT inducing treatments show a positive correlation with histone deacetylase (HDAC) related gene sets (Table 1, HDAC), a feature of chromatin rearrangement, thus suggesting that MAT inducing treatments impact on gene transcriptional regulation. Importantly, in all MAT inducing treatments we found an important positive correlation with the HOXA5 controlled pathways (Table 1, HOXA5). HOXA5 is a transcription factor with a crucial role during morphogenesis and tumourigenesis [35]. Although it has not yet been involved in MAT and studies on its role in control of motility are still at their infancy, HOXA5 has been implicated in repression of EMT through regulation of ZEB1 or Snail [36-38]. These indications are in keeping with our observation that MAT induces a repression of the mesenchymal phenotype (Table 1, epithelial-mesenchymal transition).

\section{MAT promotes an increase in stem cell markers, self-renewal of melanoma cells, tumour growth in nude mice}

To further investigate the link between stemness and MAT, we decided to analyse whether EphA2 or RacN17 overexpression, treatment with Rho activator or Ilomastat are able to further enhance the stemness of melanoma cells. Flow cytometry analysis of Hs294T cells reveals that all treatments inducing MAT enhance expression of $\mathrm{CD} 20$ and $\mathrm{CD} 133$, established stemness markers in melanoma (Figure 3A-B). In addition, qRTPCR analysis showed increased levels of known embryonic stem cell factors like KLF4, NANOG, SOX2 and OCT4 which are involved in the maintenance of the undifferentiated state of stem cells and in the stem cell self-renewal $[39,40]$ (Figure 3C-F). In keeping with the increase of the stem cell markers, activation of MAT increases the clonogenic potential of Hs294T cells, assessed by melanospheres formation assay and P1 melanospheres development (Figure 3G-H). The ability to form melanospheres is in keeping with anchorage independence and resistance to anoikis of Hs294T melanoma cells. We also confirm the link between MAT and stemness in a different cellular system, i.e. PC3 prostate carcinoma cells undergoing MAT in response to contact with endothelial cells [41]. Again, in MAT undergoing cells we observed an increase in stem cell markers, as well as an increase of the clonogenic potential (Additional file 2: Figure S1). These data confirm that MAT can induce a stem cell phenotype in different tumour types, independently of the MAT inducing stimuli.

EphA2 expression is a common event during activation of MAT. In keeping with this, both Ilomastat and Rho activator induce EphA2 expression in melanoma cells (Figure 4A). For this reason, between the different treatments able to induce amoeboid motility, we selected EphA2 overexpressing cells to perform in vivo experiments. To test whether MAT could promote tumour growth in vivo, we compared the tumour initiating 
A

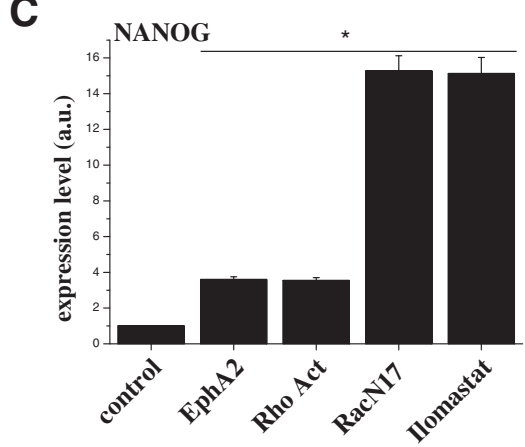

E

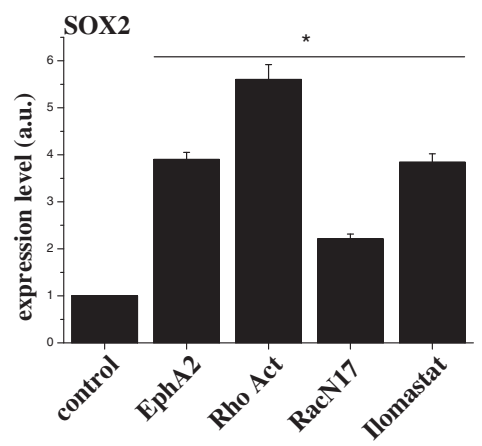

G

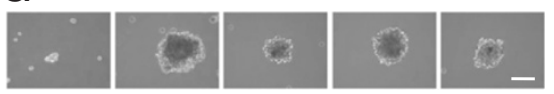

Control EphA2 RhoAct RacN17 llomastat

\begin{tabular}{|l|l|l|l|l|l|}
\hline $\begin{array}{l}\text { Clones } \mathrm{n}^{\circ} \\
\text { /field }\end{array}$ & $2.5 \pm 0.13$ & $5.2 \pm 0.20$ & $4.5 \pm 0.22$ & $5.7 \pm 0.29$ & $4.4 \pm 0.25$ \\
\hline
\end{tabular}

B

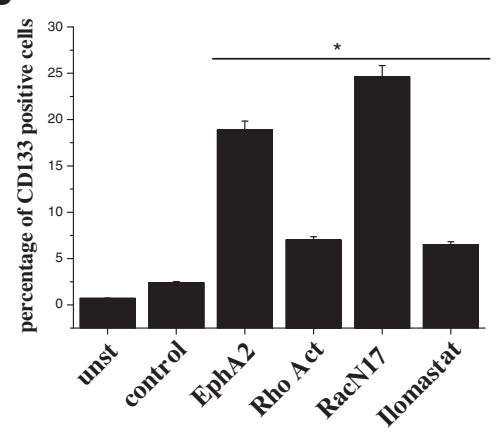

D

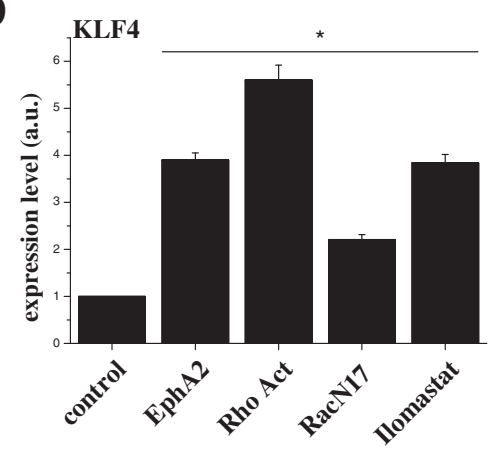

$\mathbf{F}$

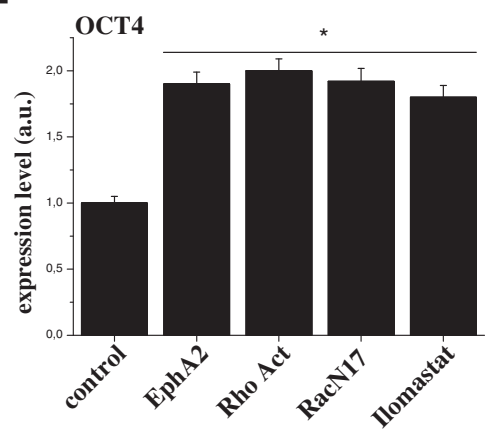

H

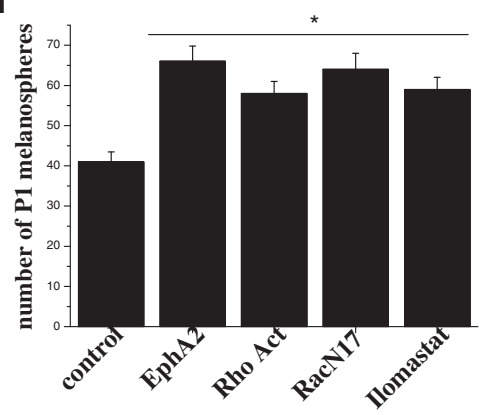

Figure 3 Cells overexpressing EphA2 or RacN17, treated with Rho activator or ilomastat promote MAT and induce an increase in stem cell markers and clonogenic potential. A-B) Hs294T, EphA2 or RacN17 transfected cells were serum starved for 48 h, for llomastat treatment cells were serum starved for $48 \mathrm{~h}$ in the presence of $50 \mu \mathrm{mol} / \mathrm{L}$ llomastat, for Rho activation treatment cells were serum starved for $48 \mathrm{~h}$ and then stimulated with $1 \mathrm{U} / \mathrm{ml}$ Calpeptin for $2 \mathrm{~h}$ at $37^{\circ} \mathrm{C}$. Cells were then analysed for expression of the cell-surface markers CD20 and CD133 by means of cytometry. The CD20 or and CD133-positive populations were plotted. The results are representative of three experiments with similar results. Student t-test, ${ }^{*} p<0.001$ treatments vs control. C-F) Cells were treated as in A). Total RNA was extracted and NANOG, KLF4, SOX2 and OCT4 mRNA expression level was analyzed by qRT-PCR. Results are representative of three experiments with similar results. Student t-test, ${ }^{*} p<0.005$ treatments vs control. G) Representative images of clones obtained from cells treated as in $\mathbf{A}$ ) after 10 days of culturing at clonal densities. Bar, $100 \mu \mathrm{m}$. Clones were photographed, counted and the mean \pm SD of clones number/field is reported. H) P1 individual spheres, derived from dissociated single melanospheres, were counted and the bar graphs obtained from triplicate experiments are shown. Student t-test, ${ }^{*} p<0.005$. 


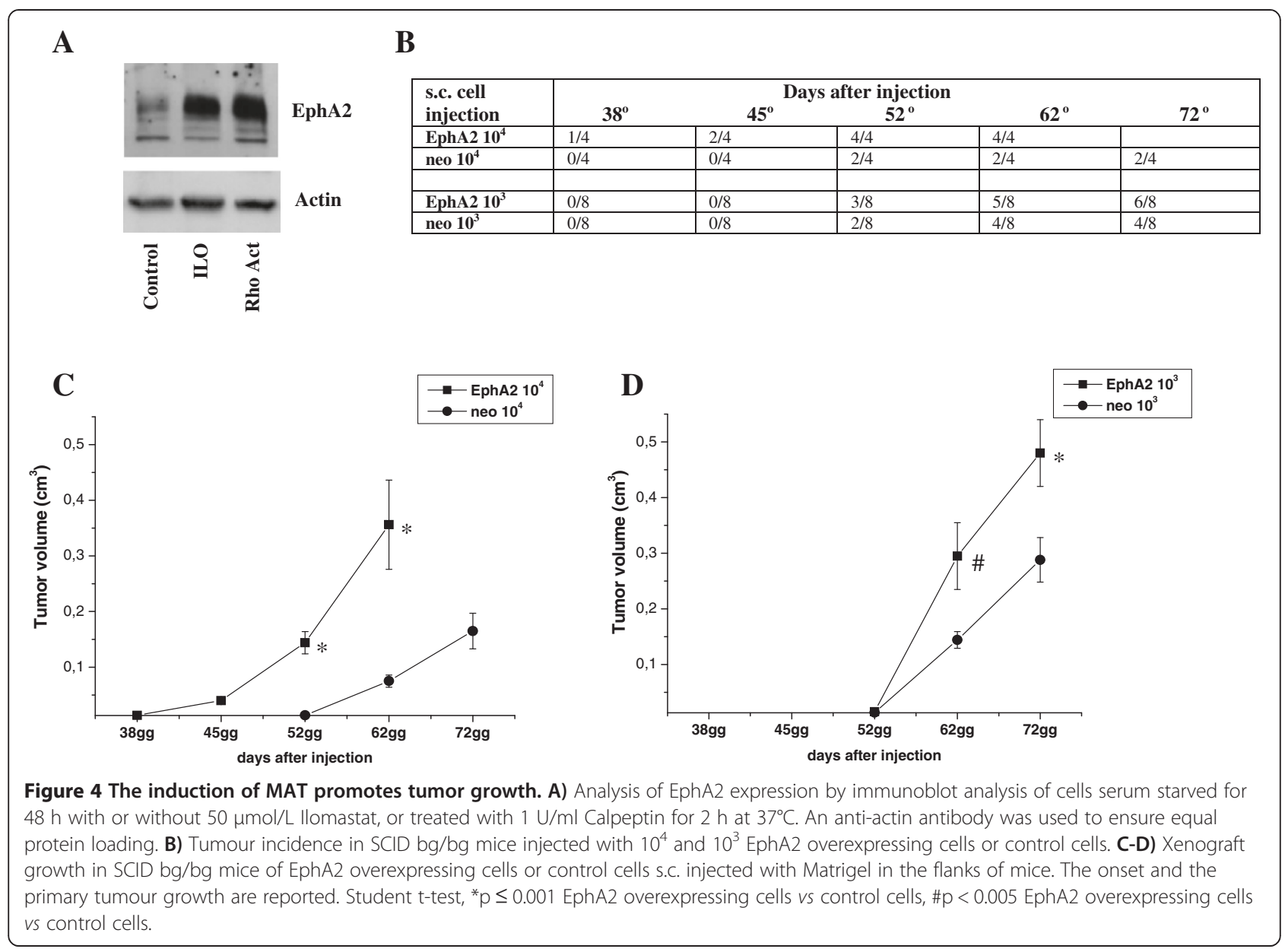

capacities of control melanoma cells and EphA2 overexpressing cells after s.c. injection into SCID-bg/bg mice. At lower concentration $\left(10^{3}\right.$ cells $)$ EphA2 influences the rate of tumour growth and at higher concentration $\left(10^{4}\right.$ cells) both the onset and the growth of tumour are influenced by EphA2 overexpression (Figure 4C-D), thus demonstrating that the induction of MAT, in parallel with an enrichment of stem cell traits in Hs294T melanoma cells, drives an increase in tumourigenesis.

\section{Conclusion}

In conclusion, MAT is likely to be an epigenetic invasive programme, hierarchically succeeding EMT, which further strengthens the stem-like and clonogenic features of cancer cells. For this reason, before fixing the concept that stemness is due to EMT engagement [16,17,23,29,36-38, 42,43 ], it should be more correct to correlate stemness to enhanced plasticity in cells motility, a wider concept including EMT and MAT. Pharmacological strategies aimed at blocking only EMT are therefore destined to collide with the enormous adaptive and plastic features of cancer cells and should be revised by including MAT as an additional target of anti metastatic treatments.

\section{Methods}

Materials

Unless otherwise specified all reagents were obtained from Sigma. Anti EphA2 antibodies were from Upstate Biotechnology Inc. The invasion chambers were from Corning Costar. The Matrigel Matrix, anti Rac1 antibody were from RD System. Ilomastat was from Chemicon International. RNeasy Plus Mini kit was from Qiagen. Type I collagen, the FITC mouse anti human CD44 (clone G44-26) and PE mouse anti human CD24 (clone ML5) antibodies were from BD Bioscience (San Jose, CA, USA). CD133 and CD20 antibodies were from Abcam. Anti mouse Alexa 488 antibody was from Molecular Probes. The Rho activator was from Cytoskeleton. Magnetic Dynabeads CD31 for endothelial cell separation was obtained from Invitrogen.

\section{Cell culture and transfection}

Hs294T human melanoma cells and prostate cancer cells (PC3) were purchased from ATCC and cultured in DMEM supplemented with $10 \% \mathrm{FCS}$ at $37^{\circ} \mathrm{C}$ in $5 \% \mathrm{CO}_{2}$ humidified atmosphere. Endothelial progenitor cells (EPCs) have been isolated from human umbilical cord 
blood as previously described [44,45]. EPCs were cultured on gelatin 1\% coated dishes in EGM-2 medium (Lonza). Hs294T cells were transfected with RacN17 (a dominant negative mutant of Rac1) or EphA2 constructs using Lipofectamine 2000 (Invitrogen) according to manifacturer's instructions.

\section{Analysis of cell morphology in 3D matrix}

Cells were labeled by $5 \mu \mathrm{mol} / \mathrm{L}$ Cell Trace CFSE (Invitrogen) for $30 \mathrm{~min}$ at $37^{\circ} \mathrm{C}$. Cells were then detached by Accutase (Sigma), washed and incorporated into 3-dimensional (3D) collagen I lattice $(1.67 \mathrm{mg} / \mathrm{mL}$ rat tail collagen I). After $5 \mathrm{~h}$, photographs were taken under confocal microscopy (Leica-SP5 system).

\section{Cell viability assay}

$10^{5}$ cells were detached using Accutase (Sigma) and suspended with $100 \mu \mathrm{l}$ of the Muse ${ }^{\mathrm{Tx}}$ Annexin V \& Dead Cell Reagent (Millipore) according to manufacturer's instructions. After $20 \mathrm{~min}$, the percentage of apoptotic cells was analyzed by the Muse ${ }^{\mathrm{mt}}$ Cell Analyzer (Millipore).

\section{RhoA or Rac1 activity assay}

Cells were directly lysed in RIPA buffer, the lysates were clarified by centrifugation and RhoA-GTP or Rac-GTP were quantified. Briefly, lysates were incubated with 10 $\mu \mathrm{g}$ Rhotekin-GST fusion protein (Becton Dickinson) or p21 activated kinase (PAK)-GST fusion protein, both absorbed on glutathione Sepharose beads for $1 \mathrm{~h}$ at $4^{\circ} \mathrm{C}$. Immunoreactive RhoA or Rac1 were then quantified by western blot analysis. Lysates were normalised for RhoA or Rac1 content by immunoblot.

\section{Western blot analysis}

$1 \times 10^{6}$ cells were lysed for $20 \mathrm{~min}$ on ice in $500 \mu \mathrm{l}$ of complete radioimmunoprecipitation assay (RIPA) lysis buffer [50 mM Tris- $\mathrm{HCl}$ (pH 7.5), $150 \mathrm{mM} \mathrm{NaCl}, 1 \%$ NP40, 2 mM EGTA, $1 \mathrm{mM}$ sodiumorthovanadate, $1 \mathrm{mM}$ phenylmethylsulfonylfluoride, $10 \mu \mathrm{g} / \mathrm{ml}$ aprotinin, $10 \mu \mathrm{g} /$ $\mathrm{ml}$ leupeptin]. Lysates were clarified by centrifuging, separated by SDS-PAGE, and transferred onto nitrocellulose. The immunoblots were incubated in $3 \%$ bovine serum albumin, $10 \mathrm{mM}$ Tris- $\mathrm{HCl}(\mathrm{pH}$ 7.5), $1 \mathrm{mM}$ EDTA and $0.1 \%$ Tween 20 for $1 \mathrm{~h}$ at room temperature and were probed first with specific antibodies and then with secondary antibodies.

\section{Cell co-cultures}

PC3 were cultured with EPCs (1:1 ratio) in EGM-2 serum-free medium for $48 \mathrm{~h}$. PC3 cells alone were plated as a control. At the end of the co-culture, cells were separated using magnetic Dynabeads CD31 (Invitrogen) according to manufacturer's instructions.

\section{Invasion assay}

Cells were serum starved for $48 \mathrm{~h}$ and then $6 \times 10^{4}$ cells were seeded onto Matrigel-precoated Boyden chamber $(8 \mathrm{~mm}$ pore size, $6.5 \mathrm{~mm}$ diameter, $12.5 \mu \mathrm{g}$ Matrigel/filter) with or without $50 \mu \mathrm{M}$ Ilomastat. In the lower chamber, complete medium was added as chemo attractant. Following $24 \mathrm{~h}$ of incubation, the inserts were removed and the non invading cells on the upper surface were removed with a cotton swab. The filters were then stained using the Diff-Quick kit (BD Biosciences) and photographs of randomly chosen fields are taken.

\section{Gelatin zymography}

Serum free medium from monolayer of cells was collected and $20 \mu \mathrm{l}$ were added to sample buffer (SDS 0.4\%, $2 \%$ glycerol, $10 \mathrm{mM}$ Tris-HCl, pH 6.8, 0.001\% bromphenol blue). The sample were run on a $10 \%$ SDS gel containing $0.1 \%$ gelatin. After electrophoresis the gel was washed twice with $2.5 \%$ Triton X-100 and once with reaction buffer ( $50 \mathrm{mM}$ Tris- $\mathrm{HCl}, \mathrm{pH} 7.5,200 \mathrm{mM} \mathrm{NaCl}$, $5 \mathrm{mM} \mathrm{CaCl}_{2}$ ). The gel was incubated over night at $37^{\circ} \mathrm{C}$ with freshly added reaction buffer and stained with Laemli Comassie blue solution. Areas of gelatinase activity appear as clear bands against a dark background.

\section{Gene expression profiling}

Hs294T were serum starved for $48 \mathrm{~h}$ and in the presence of $50 \mu \mathrm{mol} / \mathrm{L}$ Ilomastat or serum starved for $48 \mathrm{~h}$ and treated with the Rho activator Calpeptin $1 \mathrm{U} / \mathrm{ml}$ for the last $2 \mathrm{~h}$ of incubation. EphA2-overexpressing Hs294T cells were serum starved for $48 \mathrm{~h}$. Total RNA was isolated from Hs294T cells using RNeasy Plus Mini kit (Qiagen). Duplicate sample from 2 independent experiments were hybridized onto Human AffymetrixHuGene St 1.0 GeneChip array (Affymetrix) by Microarray Unit Cogentech (Milan, Italy). Data were normalised by RMA (Robust Multichip Analysis) algorithm using the affy package of Bioconductor/R. Microarray data have been uploaded in GEO (GSE52246). Class comparison between treated and controls samples was performed using a moderated t-test as implemented in the limma package of Bioconductor/R. Gene set enrichment analysis was performed using the GSEA v2.0 [24] on the pre-ranked gene list by applying the $\mathrm{t}$-test statistics as ranking criteria. Both $\mathrm{C} 2$ (canonical pathways and signatures from the literature) and C5 (gene ontology terms) gene set collections from the MSigDB database (http://www.broadinstitute.org/gsea/msigdb) were tested for enrichment and gene sets with FDR $<1 \%$ were considered significantly enriched. Several biofunctions, each supported by multiple enriched gene sets were identified and reported in Table 1 and a complete list of the gene sets is reported in Additional file 1: Table S1. 


\section{Flow cytometer analysis}

To determine the surface expression of CD20, CD133, CD44 and CD24 $10^{6}$ cells were detached nonenzymatically with $2.5 \mathrm{mM}$ EDTA and incubated with the antibodies according to manufacturer's instructions in PBS containing $1 \%$ BSA for $1 \mathrm{~h}$ at $4^{\circ} \mathrm{C}$. After washing with $\mathrm{PBS} / 1 \%$ BSA cells were incubated with Alexa 488 labelled anti mouse antibodies for $30 \mathrm{~min}$ at $4^{\circ} \mathrm{C}$. Upon washing, a flow cytometer analysis was performed.

\section{Real time RT-PCR}

Total RNA from Hs294T melanoma cells was extracted using RNeasy (Qiagen) according to the manufacturer instructions. Strands of cDNA were synthesized using a high capacity cDNA reverse transcription kit (Applied Biosystem) using $1 \mu \mathrm{g}$ of total RNA. For quantification of mRNA expression, Real-Time PCR, using Power SYBR green dye (Applied Biosystem) was done on a 7500 Fast Real Time PCR system (Applied Biosystem). The primers were NANOG: $5^{\prime}$-ACCTTGGCTGCCGTCT CTGG-3' (forward), 5' -AGCAAAGCCTCCCAATCCCAA ACA-3' (reverse); KLF4: 5'-GCAGCCACCTGGCGAGTC TG-3' (forward), 5'-CCGCCAGCGGTTATTCGGGG-3' (reverse); SOX2 5'-GAGCTTTGCAGGAAGTTTGC-3' (forward), 5'-GCAAGAAGCCTCTCCTTGAA-3' (reverse); OCT4: 5'-TTTTGGTACCCCAGGCTATG-3' (forward), 5'-GCAGGCACCTCAGTTTGAAT-3' (reverse). Data were normalized to those obtained with Glyceraldehyde3-phosphate deydrogenase primers. Results (mean $\pm \mathrm{SD}$ ) are the mean of three different experiments.

\section{Prostaspheres/melanospheres formation and clonogenicity assay}

Cells were detached using Accutase (Sigma). For prostaspheres and melanospheres formation, single cells were plated at 150 cells $/ \mathrm{cm}^{2}$ on low attachment $100 \mathrm{~mm}$ plate (Corning) in DMEM/F12 (Invitrogen, Carlsbad, CA, USA) supplemented with B27 and N2 (Invitrogen), $5 \mu \mathrm{g} / \mathrm{ml}$ insulin, $20 \mathrm{ng} / \mathrm{ml} \mathrm{bFGF}$ and $20 \mathrm{ng} / \mathrm{ml}$ EGF for prostaspheres or supplemented with $\mathrm{N} 2,0.6 \%$ glucose, $20 \mu \mathrm{g} / \mathrm{ml}$ insulin, $10 \mathrm{ng} / \mathrm{ml} \mathrm{bFGF}$ and $100 \mathrm{ng} / \mathrm{ml}$ EGF for melanospheres. Cells were grown under these conditions for 10 days and then prostaspheres and melanospheres were photographed. For the evaluation of self-renewal, a single melanosphere was dissociated in single cells with Accutase, and a diluition of one cell per well into 96-well low attachment plates was performed in order to isolate individual P1 melanospheres. Single-cell cloning was confirmed by microscopic analysis, and single clones were counted.

\section{In vivo experiments}

Xenograft experiments were performed in agreement with national guidelines and approved by the ethical committee of Animal Welfare Office of Italian Work
Ministry and conform to the legal mandates and Italian guidelines for the care and maintenance of laboratory animals. 6-8 weeks old male SCID-bg/bg mice (Charles River Laboratories International, Inc., Wilmington, MA, USA) were injected subcutaneously (s.c.), both in the right and left lateral flanks, with cells mixed in a 1:1 volume ratio with Matrigel, in a final volume of $200 \mu \mathrm{l}$. Animals were monitored, tumour size was measured by a caliper and tumour volumes determined by the length $(L)$ and the width $(W): V=\left(L W^{2}\right) / 2$.

\section{Additional files}

Additional file 1: Table S1. GSEA analysis output. Gene Set Enrichment Analysis was performed with C2 and C5 collections of gene sets as described in the Methods section.

Additional file 2: Figure S1. MAT promotes stemness in prostate cancer cells. A) PC3 cells were co-cultured or not with EPC for $48 \mathrm{~h}$ in order to induce MAT, then photographs were taken, the arrow shows a representative cell that has acquired a rounded morphology. Bar, 50 um. B) PC3 cells were treated as in A), after separation, PC3 cells were analysed for expression of the cell-surface marker FITC-CD44 and PE-CD24 and CD133 by means of cytometry. The CD44 $4^{\text {high }} /$ CD24 $4^{\text {low }}$ or CD133-positive populations were plotted. Results shown are representative of three experiments. Student t-test, ${ }^{*} p<0.001$ PC3 in co-culture vs PC3 alone. C) Representative images of clones obtained from PC3 cells or PC3 co-cultured with EPC after 20 days of culturing at clonal densities. Bar, $100 \mu \mathrm{m}$.

\section{Abbreviations}

ECM: Extra cellular matrix; EMT: Epithelial mesenchymal transition; MAT: Mesenchymal epithelial transition; MMPs: Matrix metallo proteinases.

\section{Competing interests}

The authors declare no competing interests.

\section{Authors' contributions}

$M L T, E G, L I, A M, M R$ performed the experiments; MC, PG analysed microarray data; MLT, AM and PC wrote the manuscript. All authors read and approved the final manuscript.

\section{Acknowledgements}

The work was supported by Associazione Italiana Ricerca sul Cancro (AIRC), grants \#11542 (P.G.), grants \# 8797 (P.C.), Istituto Toscano Tumori

(No. 0203607) (P.C.), by a fellowship from Fondazione Italiana Ricerca sul Cancro (FIRC) to A.M. and by Programma operativo regionale Obiettivo

"Competitività regionale e occupazione" della Regione Toscana cofinanziato dal Fondo europeo di sviluppo regionale 2007-2013 (POR CReO FESR 2007-2013).

\section{Author details}

'Department of Experimental and Clinical Biomedical Sciences, Tuscany Tumor Institute, University of Florence, viale Morgagni 50, Florence 50134, Italy. ${ }^{2}$ Department of Experimental Oncology and Molecular Medicine, Fondazione IRCCS Istituto Nazionale dei Tumori, Milan 20133, Italy. ${ }^{3}$ Center for Research, Transfer and High Education 'Study at Molecular and Clinical Level of Chronic, Inflammatory, Degenerative and Neoplastic Disorders for the Development on Novel Therapies', Florence, Italy.

Received: 8 November 2013 Accepted: 11 March 2014 Published: 1 April 2014

\section{References}

1. Friedl P, Wolf K: Tumour-cell invasion and migration: diversity and escape mechanisms. Nat Rev Cancer 2003, 3:362-374. 
2. Sahai E: llluminating the metastatic process. Nat Rev Cancer 2007, 7:737-749.

3. Yamazaki D, Kurisu S, Takenawa T: Regulation of cancer cell motility through actin reorganization. Cancer Sci 2005, 96:379-386.

4. Lah TT, Duran Alonso MB, Van Noorden CJ: Antiprotease therapy in cancer: hot or not? Expert Opin Biol Ther 2006, 6:257-279.

5. Wolf K, Mazo I, Leung H, Engelke K, von Andrian UH, Deryugina El, Strongin $A Y$, Brocker EB, Freidl P: Compensation mechanism in tumor cell migration: mesenchymal-amoeboid transition after blocking of pericellular proteolysis. J Cell Biol 2003, 160:267-277.

6. Giannoni E, Parri M, Chiarugi P: EMT and oxidative stress: a bidirectional interplay affecting tumor malignancy. Antioxid Redox Signal 2012, 16:1248-1263

7. Kalluri R, Weinberg RA: The basics of epithelial-mesenchymal transition. J Clin Invest 2009, 119:1420-1428.

8. Thiery JP: Epithelial-mesenchymal transitions in tumour progression. Nat Rev Cancer 2002, 2:442-454

9. Friedl P, Wolf K: Plasticity of cell migration: a multiscale tuning model. J Cell Biol 2010, 188:11-19.

10. Berton S, Belletti B, Wolf K, Canzonieri V, Lovat F, Vecchione A, Colombatti A, Fried $P$, Baldassarre $G$ : The tumor suppressor functions of p27(kip1) include control of the mesenchymal/amoeboid transition. Mol Cell Biol 2009, 29:5031-5045.

11. Gadea G, de TM, Anguille C, Roux P: Loss of p53 promotes RhoA-ROCKdependent cell migration and invasion in 3D matrices. J Cell Biol 2007, 178:23-30.

12. Friedl P: Prespecification and plasticity: shifting mechanisms of cell migration. Curr Opin Cell Biol 2004, 16:14-23.

13. Parri M, Taddei ML, Bianchini F, Calorini L, Chiarugi P: EphA2 reexpression prompts invasion of melanoma cells shifting from mesenchymal to amoeboid-like motility style. Cancer Res 2009, 69:2072-2081.

14. Taddei ML, Parri M, Angelucci A, Onnis B, Bianchini F, Giannoni E, Taddei ML, Parri M, Angelucci A, Onnis B, Bianchini F: Kinase-dependent and -independent roles of EphA2 in the regulation of prostate cancer invasion and metastasis. Am J Pathol 2009, 174:1492-1503.

15. Taddei ML, Parri M, Angelucci A, Bianchini F, Marconi C, Giannoni E, Rauge G, Bologna M, Calorini L, Chiarugi P: EphA2 induces metastatic growth regulating amoeboid motility and clonogenic potential in prostate carcinoma cells. Mol Cancer Res 2011, 9:149-160.

16. Mani SA, Guo W, Liao MJ, Eaton EN, Ayyanan A, Zhou AY, Brooks M, Reinhard F, Zhang CC, Shiptsin M, Campbell LL, Polyak K, Brisken C, Yang J, Weinberg RA: The epithelial-mesenchymal transition generates cells with properties of stem cells. Cell 2008, 133:704-715.

17. Giannoni E, Bianchini F, Masieri L, Serni S, Torre E, Calorini L, Chiarugi P: Reciprocal activation of prostate cancer cells and cancer-associated fibroblasts stimulates epithelial-mesenchymal transition and cancer stemness. Cancer Res 2010, 70:6945-6956.

18. Binda E, Visioli A, Giani F, Lamorte G, Copetti M, Pitter KL, Huse JT, Cajola L, Zanetti N, DiMeco F, De Filippis L, Mangiola A, Maira G, Anile C, De Bonis P, Reynolds BA, Pasquale EB, Vescovi AL: The EphA2 receptor drives self-renewal and tumorigenicity in stem-like tumor-propagating cells from human glioblastomas. Cancer Cell 2012, 22:765-780.

19. Sanz-Moreno V, Gadea G, Ahn J, Paterson H, Marra P, Pinner S, Sahai E, Marshall CJ: Rac activation and inactivation control plasticity of tumor cell movement. Cell 2008, 135:510-523.

20. Ahn J, Sanz-Moreno V, Marshall CJ: The metastasis gene NEDD9 product acts through integrin beta3 and Src to promote mesenchymal motility and inhibit amoeboid motility. J Cell Sci 2012, 125:1814-1826.

21. Sanz-Moreno V, Gaggioli C, Yeo M, Albrengues J, Wallberg F, Viros A, Hooper S, Mitter R, Feral CC, Cook M, Larkin J, Marais R, Maneguzzi G, Sahai E, Marshall CJ: ROCK and JAK1 signaling cooperate to control actomyosin contractility in tumor cells and stroma. Cancer Cell 2011, 20:229-245.

22. Nieto MA: The snail superfamily of zinc-finger transcription factors. Nat Rev Mol Cell Biol 2002, 3:155-166.

23. Nieto MA, Cano A: The epithelial-mesenchymal transition under control: global programs to regulate epithelial plasticity. Semin Cancer Biol 2012, 22(5-6):361-368.

24. Subramanian A, Tamayo P, Mootha VK, Mukherjee S, Ebert BL, Gillette MA Paulovich A, Pomeroy SL, Golub TR, Lander ES, Mesirov JP: Gene set enrichment analysis: a knowledge-based approach for interpreting genomewide expression profiles. Proc Natl Acad Sci USA 2005, 102:15545-15550.

25. Brabek J, Mierke $C T$, Rosel D, Vesely P, Fabry B: The role of the tissue microenvironment in the regulation of cancer cell motility and invasion. Cell Commun Signal 2010, 8:22.
26. Sabeh F, Shimizu-Hirota R, Weiss SJ: Protease-dependent versus -independent cancer cell invasion programs: three-dimensional amoeboid movement revisited. J Cell Biol 2009, 185:11-19.

27. Taddei ML, Giannoni E, Comito G, Chiarugi P: Microenvironment and tumor cell plasticity: an easy way out. Cancer Lett 2013, 341:80-96.

28. Giannoni E, Buricchi F, Grimaldi G, Parri M, Cialdai F, Taddei ML, Raugei G, Ramponi G, Chiarugi P, Raugei G, Ramponi G, Chiarugi P: Redox regulation of anoikis: reactive oxygen species as essential mediators of cell survival. Cell Death Differ 2008, 15:867-878

29. Giannoni E, Bianchini F, Calorini L, Chiarugi P: Cancer associated fibroblasts exploit reactive oxygen species through a proinflammatory signature leading to epithelial mesenchymal transition and stemness. Antioxid Redox Signal 2011, 14:2361-2371.

30. Semenza GL: Hypoxia-inducible factors: mediators of cancer progression and targets for cancer therapy. Trends Pharmacol Sci 2012, 33:207-214.

31. Chen HY, White E: Role of autophagy in cancer prevention. Cancer Prev Res (Phila) 2011, 4:973-983.

32. Chen S, Rehman SK, Zhang W, Wen A, Yao L, Zhang J: Autophagy is a therapeutic target in anticancer drug resistance. Biochim Biophys Acta 2010, 1806:220-229

33. Orlotti NI, Cimino-Reale G, Borghini E, Pennati M, Sissi C, Perrone F, Palumbo M, Diadone MG, Folini M, Zaffaroni N: Autophagy acts as a safeguard mechanism against G-quadruplex ligand-mediated DNA damage. Autophagy 2012, 8:1185-1196.

34. Hanahan D, Weinberg RA: Hallmarks of cancer: the next generation. Cell 2011, 144:646-674.

35. Boucherat O, Guillou F, Aubin J, Jeannotte L: Hoxa5: a master gene with multifaceted roles. Med Sci (Paris) 2009, 25:77-82.

36. Asuthkar S, Stepanova V, Lebedeva T, Holterman AL, Estes N, Cines DB, Rao $J S$, Gondi CS: Multifunctional roles of urokinase plasminogen activator (UPA) in cancer stemness and chemoresistance of pancreatic cancer. Mol Biol Cell 2013, 24:2620-2632.

37. Azmi AS, Bollig-Fischer A, Bao B, Park BJ, Lee SH, Yong-Song G, Dyson G, Reddy CK, Sarkar FH, Mohammad RM: Systems analysis reveals a transcriptional reversal of the mesenchymal phenotype induced by SNAIL-inhibitor GN-25. BMC Syst Biol 2013, 7:85.

38. Cieply B, Farris J, Denvir J, Ford HL, Frisch SM: Epithelial-mesenchymal transition and tumor suppression are controlled by a reciprocal feedback loop between ZEB1 and grainyhead-like-2. Cancer Res 2013, 73:6299-6309.

39. Santini R, Vinci MC, Pandolfi S, Penachioni JY, Montagnani V, Olivito B, Gattai R, Pimpineli N, Gerlini L, Borgognoni L, Stecca B: Hedgehog-GLI signaling drives self-renewal and tumorigenicity of human melanoma-initiating cells. Stem Cells 2012, 30:1808-1818

40. Zimmerer RM, Korn P, Demougin P, Kampmann A, Koke muller H, Eckardt AM, Gellrich NC, Tavassol F: Functional features of cancer stem cells in melanoma cell lines. Cancer Cell Int 2013, 13:78.

41. Giannoni E, Taddei ML, Parri M, Bianchini F, Santosuosso M, Grifantini R, Fibbi G, Mazzanti B, Calorini L, Chiarugi P: EphA2-mediated mesenchymalamoeboid transition induced by endothelial progenitor cells enhances metastatic spread due to cancer-associated fibroblasts. J Mol Med (Berl) 2013, 91:103-115.

42. Sanchez-Tillo E, Liu Y, De BO, Siles L, Fanlo L, Cuatrecasas M, Darling DS, Dean DC, Castells A, Podtigo A: EMT-activating transcription factors in cancer: beyond EMT and tumor invasiveness. Cell Mol Life Sci 2012, 69:3429-3456.

43. Thiery JP, Acloque H, Huang RY, Nieto MA: Epithelial-mesenchymal transitions in development and disease. Cell 2009, 139:871-890.

44. Margheri F, Chilla A, Laurenzana A, Serrati S, Mazzanti B, Saccardi R, Santosuosso M, Danza G, Sturli N, Rosati F, Magnelli L, Papucci L, Calorini L, Bianchini F, Del Rosso M, Fibbi G: Endothelial progenitor cell-dependent angiogenesis requires localization of the full-length form of UPAR in caveolae. Blood 2011, 118:3743-3755.

45. Yoder MC, Mead LE, Prater D, Krier TR, Mroueh KN, Li F, Krasich R, Temm CJ, Prchal JT, Ingram DA: Redefining endothelial progenitor cells via clonal analysis and hematopoietic stem/progenitor cell principals. Blood 2007, 109:1801-1809.

doi:10.1186/1478-811X-12-24

Cite this article as: Taddei et al:: Mesenchymal to amoeboid transition is associated with stem-like features of melanoma cells. Cell Communication and Signaling 2014 12:24. 